\title{
XMM-Newton observations of Nova LMC 2000^
}

\author{
J. Greiner ${ }^{1}$, M. Orio ${ }^{2,3}$, and N. Schartel ${ }^{4}$ \\ 1 Max-Planck-Institut für extraterrestrische Physik, 85741 Garching, Germany \\ 2 INAF - National Institute for Astrophysics, Osservatorio Astronomico di Torino, Strada Osservatorio 20, \\ 10025 Pino Torinese, Italy \\ 3 Department of Astronomy, 475 N. Charter Str., Madison WI 53706, USA \\ 4 XMM-Newton Science Operations Centre, ESA, Villafranca del Castillo, PO Box 50727, 28080 Madrid, Spain
}

Received 28 February 2003 / Accepted 18 April 2003

\begin{abstract}
We report on three X-ray observations of Nova LMC 2000 with XMM-Newton at 17, 51 and 294 days after the maximum, respectively. X-ray spectral fits show a concordant decrease of the absorbing column and the X-ray luminosity. No supersoft X-ray emission is detected. The mass of the ejected shell is determined to be (less than) $7.5 \times 10^{-5} M_{\odot}$. Though data are sparse, one interesting correlation becomes visible: sources with a long-duration supersoft X-ray phase have shorter orbital periods than those with short or no supersoft X-ray phase. This can be understood considering that (i) enough matter has to be accreted in order to ignite the $\mathrm{H}$ burning, and (ii) that $\mathrm{H}$ burning ceases when the mass of the remaining material (after shell ejection and burning) drops below a certain limit under which the temperature at the bottom of the envelope is too low for the shell burning to compensate the energy loss from the surface.
\end{abstract}

Key words. stars: individual: N LMC 2000 - stars: mass-loss - stars: novae, cataclysmic variables - X rays: stars stars: binaries: close

\section{Introduction}

Nova LMC 2000 was discovered in mid-July 2000 (Liller 2000). Early observations between June 29.68 and July 1.68 have detected the nova, but no brightness estimates are available (Bond \& Kilmartin 2000), since all images except the first one on June 29.68 show the nova to be saturated (Bond, priv. comm.). This leaves the exact time and magnitude of the maximum unknown except for the constraint that it was still rising on June 29.68 (Bond, priv. comm.). But even if the observed $m_{\mathrm{V}}=11.2$ mag on 12 July 2000 was the maximum, with a corresponding to $M_{\mathrm{V}}=-7.5 \mathrm{mag}$, Nova LMC 2000 was among the $20 \%$ brightest novae.

Optical spectroscopy on 15 July 2000 shows emission lines of the Balmer series, several Fe multiplets and Na I D, suggesting a "Fe II" nova about one week after maximum (Duerbeck $\&$ Pompei 2000). If this time estimate is correct, the maximum of the nova could have been $m_{\mathrm{V}}=10.5$ mag (backwards extrapolation to 8 July 2000) corresponding to $M_{\mathrm{V}}=-8.2 \mathrm{mag}$.

Spectroscopy of this ONeMg nova (Shore 2002) also revealed weak absorption components in the Balmer lines at $1900 \mathrm{~km} \mathrm{~s}^{-1}$ on July 15 (Duerbeck \& Pompei 2000), and

\footnotetext{
Send offprint requests to: J. Greiner, e-mail: jcg@mpe.mpg.de

* Partly based on observations collected at the European Southern Observatory, Chile; under proposal 66.D-0391.
}

a FWZI of the Balmer lines on July 14 and 15 of $2200 \pm$ $250 \mathrm{~km} \mathrm{~s}^{-1}$ (Hearnshaw \& Yan Tse 2000). Spectra obtained with HST on August 19/20, 2000, strongly resemble those of the fast nova V382 Vel $\left(t_{3}=9\right.$ days; Della Valle et al. 2002) at 2 months after visual maximum (Shore et al. 2000).

Only 4 out of about 100 classical novae observed with ROSAT have been found to exhibit a supersoft phase (see Orio \& Greiner 1999; Orio et al. 2002). However, theoretically one expects that each nova should pass through a phase of soft X-ray emission during the later stages of decline (e.g. MacDonald et al. 1985): during the post-maximum stage, at constant bolometric luminosity, the photosphere progressively retreats as the residual hydrogen envelope is depleted, and the effective temperature rises up to the soft X-ray region. It is pretty much unclear yet what determines the appearance of supersoft X-ray emission. It has been argued (Truran 2002) that the onset of the supersoft phase is the same for all novae, about 6-8 months, and that the duration of the supersoft X-ray phase is the burning timescale of the white dwarf, i.e. proportional to the mass of the white dwarf (Truran \& Glasner 1995; Vanlandingham et al. 2001). While the first two supersoft novae, GQ Mus (Ögelman et al. 1993) and V1974 Cyg (Krautter et al. 1996), are consistent with this suggestion, observations of other recent novae imply that the picture is more complicated. 
Table 1. Observation log.

\begin{tabular}{|c|c|c|c|c|c|c|c|}
\hline $\begin{array}{l}\text { Observation interval } \\
\text { (UT) }\end{array}$ & $\begin{array}{c}\text { Time after } \\
\text { Max. (days) }\end{array}$ & $\begin{array}{l}\text { Expo.-time } \\
(\mathrm{ks})\end{array}$ & Rev. & $\begin{array}{c}\text { Count rate } \\
\text { (cts/ks) }\end{array}$ & $H R 1$ & $H R 2$ & $H R 3$ \\
\hline 2000-07-25 22:48-2000-07-26 03:19 & 17 & 16.26 & 115 & $5.0 \pm 0.6$ & - & $1.00 \pm 2.00$ & $0.00 \pm 0.11$ \\
\hline $2000-08-28$ 13:53-2000-08-28 16:40 & 51 & 10.00 & 132 & $21.0 \pm 1.6$ & $0.70 \pm 0.07$ & $-0.56 \pm 0.06$ & $-0.66 \pm 0.16$ \\
\hline 2001-03-29 19:10-2001-03-29 21:22 & 294 & 10.54 & 239 & $<1.0$ & - & - & - \\
\hline
\end{tabular}

(1) The hardness ratios are defined as $H R 1=(B-A) /(B+A), H R 2=(C-B) /(B+C)$ and $H R 3=(D-C) /(D+C)$, where $A(0.2-0.5 \mathrm{keV})$, $B(0.5-2.0 \mathrm{keV}), C(2.0-4.5 \mathrm{keV}), D(4.5-7.5 \mathrm{keV})$, are the counts in the given energy range.

Table 2. Spectral fit results of the July and August 2000 observations.

\begin{tabular}{lccccc}
\hline \hline Date & $\begin{array}{c}N_{\mathrm{H}} \\
\left(10^{22} \mathrm{~cm}^{-2}\right)\end{array}$ & $\begin{array}{c}k T \\
(\mathrm{keV})\end{array}$ & $\begin{array}{c}\text { Normalization } \\
\left(\mathrm{ph} \mathrm{keV}^{-1} \mathrm{~cm}^{-2} \mathrm{~s}^{-1}\right)\end{array}$ & $\begin{array}{c}\text { Flux (bolometric) } \\
\left(\mathrm{erg} \mathrm{cm}^{-2} \mathrm{~s}^{-1}\right)\end{array}$ & $\chi^{2} /$ d.o.f. \\
\hline & & \multicolumn{5}{c}{ bremsstrahlung model } \\
25/26 Jul. 2000 & $3.23 \pm 1.23$ & $199 \pm 100$ & $1.1 \mathrm{E}-05$ & $7.5 \mathrm{E}-14$ & 0.78 \\
25/26 Jul. 2000 & $7.00 \pm 2.57$ & 1.6 fixed & $3.6 \mathrm{E}-05$ & $13.0 \mathrm{E}-14$ & 1.28 \\
28 Aug. 2000 & $0.29 \pm 0.19$ & $1.6 \pm 1.2$ & $1.6 \mathrm{E}-05$ & $5.5 \mathrm{E}-14$ & 0.94 \\
& & & Raymond-Smith model & \\
25/26 Jul. 2000 & $3.40 \pm 1.10$ & $64 \pm 50.0$ & $2.4 \mathrm{E}-05$ & $6.9 \mathrm{E}-14$ & 0.79 \\
25/26 Jul. 2000 & $7.65 \pm 2.33$ & 2.1 fixed & $7.5 \mathrm{E}-05$ & $14.4 \mathrm{E}-14$ & 1.00 \\
28 Aug. 2000 & $0.20 \pm 0.15$ & $2.1 \pm 1.0$ & $2.3 \mathrm{E}-05$ & $4.5 \mathrm{E}-14$ & 0.92 \\
\hline
\end{tabular}

Here we report the results of a sequence of XMM-Newton observations of N LMC 2000 aimed at finding and characterizing the supersoft X-ray component. We also report a contemporaneous optical observation.

\section{Observations and results}

\subsection{XMM-Newton observations}

Observations of Nova LMC 2000 were performed at three occasions (see Table 1), about 17, 51 and 294 days after the likely maximum on 8 July 2000.

Throughout all observations the thin blocking filter was used. In the following, we primarily deal with EPIC-pn (Strüder et al. 2001) part of the X-ray data, and the optical monitor (OM; Mason et al. 2001) data.

\subsubsection{XMM-Newton EPIC-pn data}

After a first inspection of the X-ray data, a source detection run was done in the $0.5-7 \mathrm{keV}$ band, as well as in narrow bands to derive hardness ratios. These hardness ratios, the count rates in the full band and the $3 \sigma$ upper limit for the non-detection during the March 2001 observation are given in Table 1 and show the strong intensity and spectral variability of the X-ray emission of Nova LMC 2000.

For the spectral analysis, single and double events away from the edges of the CCD or bad pixels were extracted in a source region of $19^{\prime \prime}$, and a background region of outer diameter of $30^{\prime \prime}$.

Because of the larger number of collected photons we first fitted the X-ray spectrum obtained on 28 August 2000. Before the extraction of source and background photons, a time interval of $\sim 2$ ks was excluded because of high background. Since the spectrum lacks any soft component, we applied a bremsstrahlung and a Raymond-Smith model to the data. Both models give satisfactory fits, and the fit parameters are given in Table 2. During the observation on 28 August 2000, the unabsorbed X-ray luminosity was $2 \times 10^{34} \mathrm{erg} \mathrm{s}^{-1}(0.01-20 \mathrm{keV}$; assuming a distance of $55 \mathrm{kpc}$ ). Note that despite the (best-fit) absorbing column of $(2-3) \times 10^{21} \mathrm{~cm}^{-2}$, a supersoft component with a characteristic temperature of $30-50 \mathrm{eV}$ and a luminosity of $10^{36}-10^{38} \mathrm{erg} \mathrm{s}^{-1}$ would have been easily detectable.

We then used the same models to also fit the July 25/26 observation. However, due to the smaller number of counts, the temperature in both models is not constrained. Thus, in a second iteration, we fixed the temperature to the value as derived from the August 28, 2000 observation. The best-fit parameters of both fits are also given in Table 2 . The unabsorbed bolometric luminosity of Nova LMC 2000 during the July 25/26, 2000 observation was $(5.0 \pm 0.3) \times 10^{34}(D / 55 \mathrm{kpc})^{2} \mathrm{erg} \mathrm{s}^{-1}$, where the error includes also the difference between the two models. In a third iteration we tested a fit with the absorbing column fixed to the value of the July 2000 observation in order to check whether a temperature change could mimic the 

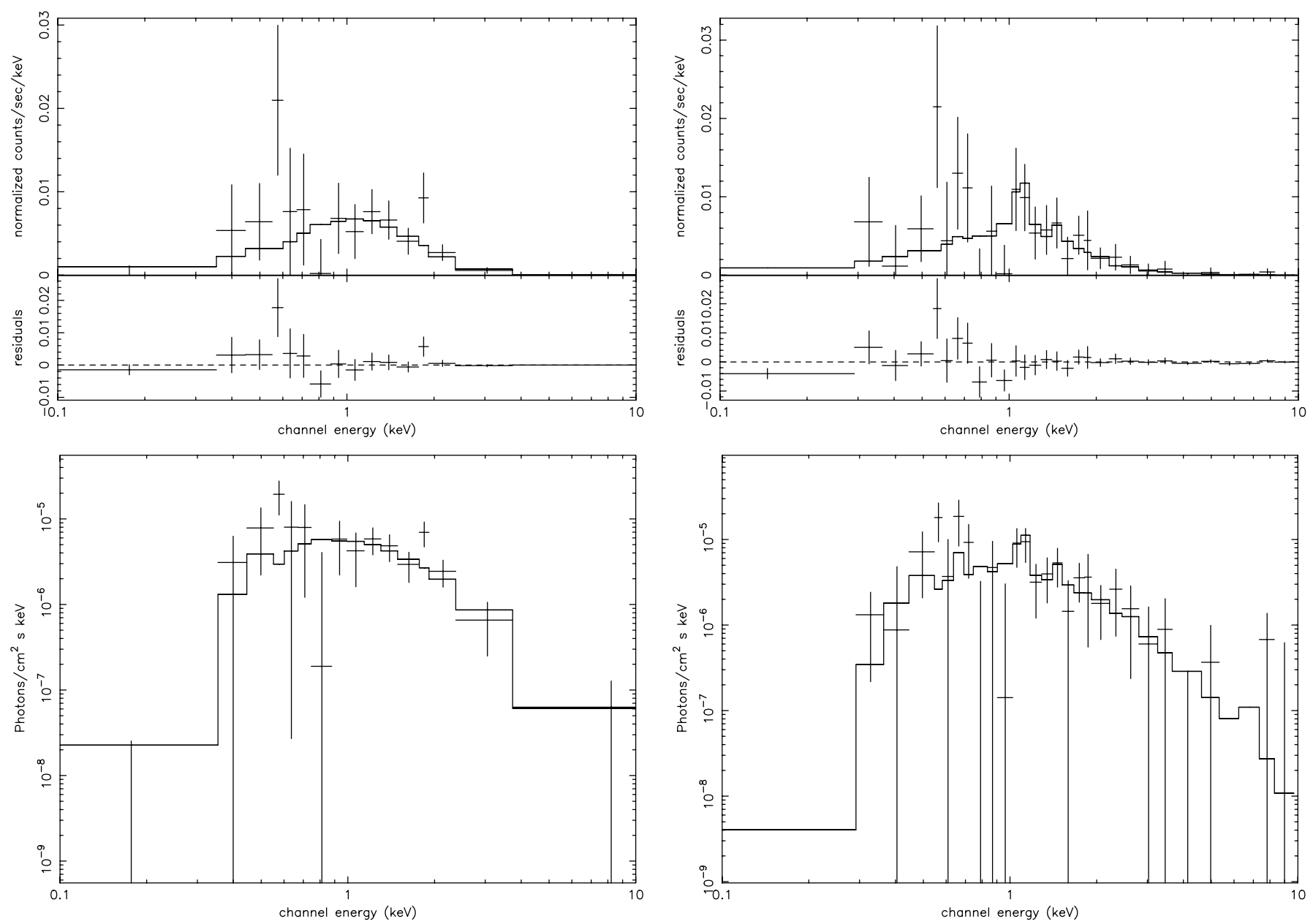

Fig. 1. X-ray spectrum of Nova LMC 2000 as observed with XMM-Newton on 28 August 2000, modelled with a bremsstrahlung (left) and Raymond-Smith model (right). The top panel shows the count rate spectrum and the residuals, while the lower panels show the unfolded photon spectrum.

Table 3. XMM/OM photometry.

\begin{tabular}{ccccc}
\hline \hline Date & Filter & $\begin{array}{c}\text { Countrate } \\
\text { cts s }^{-1}\end{array}$ & $\begin{array}{c}\text { Brightness }^{(1)} \\
\text { mag }\end{array}$ & $\begin{array}{c}\text { flux }^{(1)} \\
\text { erg cm }^{2} \text { s }^{-1} \AA^{-1}\end{array}$ \\
\hline $2000-07-25$ & $U V W 1$ & 201.4 & $11.41 \pm 0.01$ & $1.04 \mathrm{E}-13$ \\
& $U V W 2$ & 16.0 & $11.62 \pm 0.01$ & $1.24 \mathrm{E}-13$ \\
$2000-08-28$ & $V$ & 10.6 & $15.24 \pm 0.02$ & $3.05 \mathrm{E}-15$ \\
$2001-03-29$ & $U$ & 9.5 & $15.70 \pm 0.02$ & $2.24 \mathrm{E}-15$ \\
\hline
\end{tabular}

(1) Brightness and flux are given without correction for galactic, LMC and nova-intrinsic extinction, since the latter two values have uncertainties much larger than the measurement error.

variation in absorbing column. However, this does not provide an acceptable fit (reduced $\chi^{2}$ of 2.5).

For the X-ray non-detection on 29 March 2001, assuming the same spectral models and no intrinsic absorption through the ejected nova shell we derive a $3 \sigma$ upper limit for the luminosity of $L<1.1 \times 10^{33}(D / 55 \mathrm{kpc})^{2} \mathrm{erg} \mathrm{s}^{-1}$ for the case of only galactic foreground absorption $\left(7 \times 10^{20} \mathrm{~cm}^{-2}\right)$ or $L<$ $1.4 \times 10^{33}(D / 55 \mathrm{kpc})^{2} \mathrm{erg} \mathrm{s}^{-1}$ for the case of galactic foreground plus total LMC absorption $\left(15 \times 10^{20} \mathrm{~cm}^{-2}\right.$; Luks 1994).

\subsubsection{XMM-Newton Optical Monitor data}

The OM was used with a variety of filters, and also the grism was used in two occasions. In particular, we obtained (i) two $5 \mathrm{ks}$ grism 2 (visual spectrum) exposures, a sequence of 5 exposures with the $U V W 1$ filter, and a sequence of 4 exposures with the $U V W 2$ filter, each with $940 \mathrm{~s}$ ) in the first XMM-Newton observation of Nova LMC 2000 (revolution 115); (ii) a sequence of 5 exposures with the $V$ band filter with $1 \mathrm{ks}$ each, followed by a 5 ks grism 2 exposure (second XMM-Newton observation; rev. 132); (iii) a sequence of 5 exposures of $1 \mathrm{ks}$ with the $U$ band filter in the last XMM-Newton observation (rev. 239).

Unfortunately, the grism data are hardly usable since the window of the CCD was set too small. The photometry in the different filters is summarized in Table 3 . The central wavelengths for the $U V$ filters are $2910 \AA$ for the $U V W 1$ and $2120 \AA$ for the $U V W 2$, respectively.

\subsection{Optical photometry}

On 4 December 2000 we obtained three $V$ band exposures with $90 \mathrm{~s}$ exposure time each using DFOSC at the $1.5 \mathrm{~m}$ Danish 

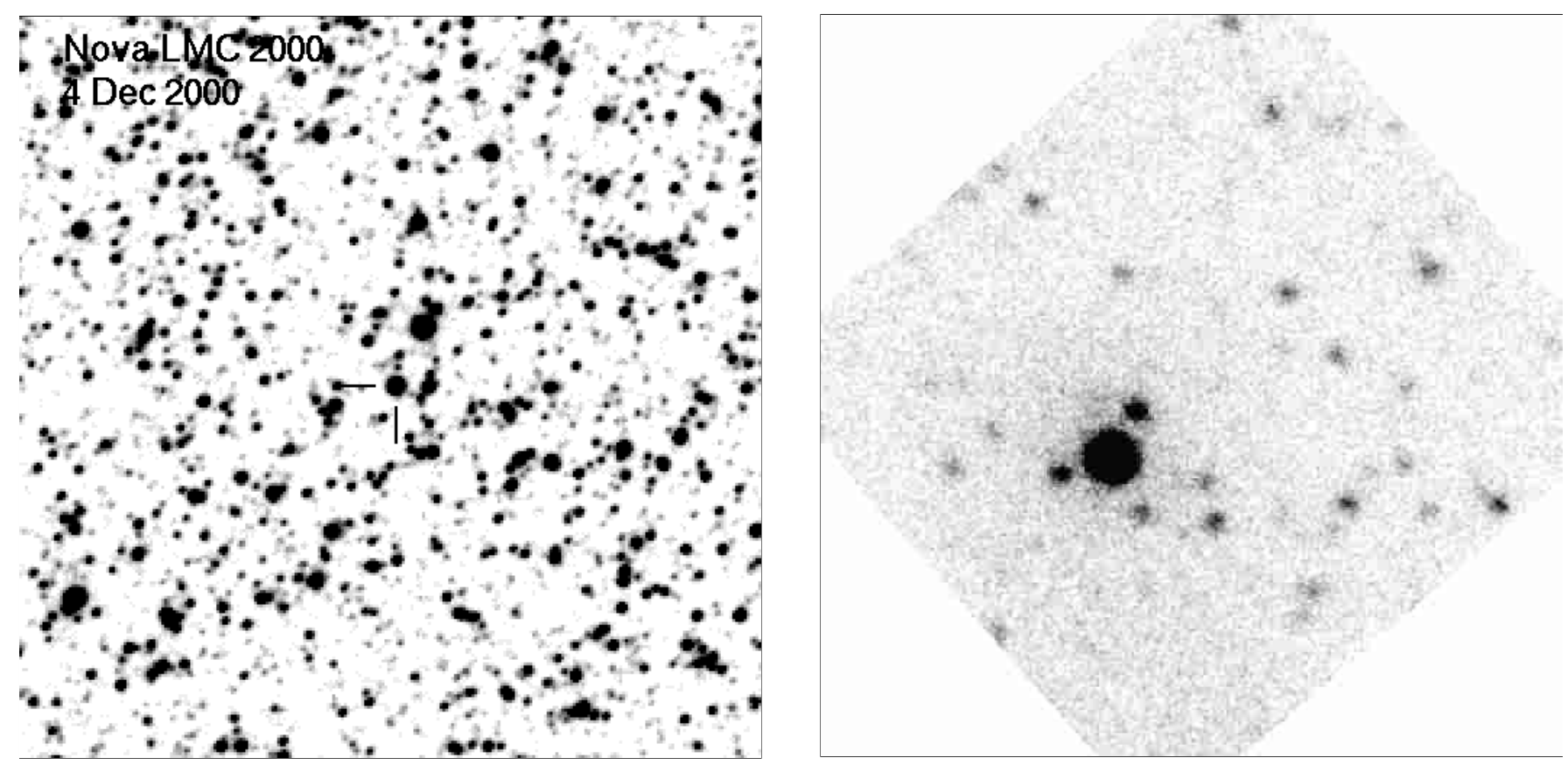

Fig. 2. Finding chart of N LMC 2000 in the $V$ band (left; marked with two dashes near the image center) and at $2120 \AA$ (right). The $V$ band image was taken on 2000 Dec. 4 with DFOSC at the $1.5 \mathrm{~m}$ Danish telescope at La Silla (ESO). We measure RA $=5^{\mathrm{h}} 25^{\mathrm{m}} 01^{\mathrm{s}} .1$ and Dec $=-70^{\circ} 14^{\prime} 17^{\prime \prime}$ (equinox 2000.0) with an error radius of $2^{\prime \prime}$. The field size is $1.7 \times 11^{\prime} 7$. The right image is the sum of 4 images with $1000 \mathrm{~s}$ exposure each, taken by the OM onboard XMM in the $U V W 2$ filter on 25/26 July 2000, where the nova is the by far brightest object in the image. The field size is $2 ! 3 \times 2 ! 3$. North is up and East to the left.

telescope at La Silla (ESO, Chile). The MAT/EEV 44-82 CCD chip with $15 \mu \mathrm{m}$ pixels has a plate scale of $0.4 /$ pixel. The seeing was $1^{\prime \prime}$. The photometric standard RU 149, observed at nearly the same airmass as N LMC 2000, was used for the absolute flux calibration. Due to the brightness of the nova and the relatively crowding-free surrounding (see Fig. 2), aperture photometry was done within the MIDAS package after standard flatfield and bias correction. We measure $V=$ $16.20 \pm 0.03 \mathrm{mag}$ independently in each of the three images for Nova LMC 2000. After adding this to the early measurements by VSNET observers (Fig. 3), a comparison with light curve models of Hachisu \& Kato suggests that Nova LMC 2000 was possibly still in the plateau phase during our optical observation on 4 December 2000.

A crude estimate of the decay rate based on the VSNET data gives $t_{2} \sim 9.0$ days and $t_{3} \sim 22$ days, with an error of \pm 2 days depending on the actual occurrence of the not observed optical maximum (where $t_{2}$ and $t_{3}$ are the times during which the brightness decays by two and three magnitudes, respectively).

\section{Discussion}

\subsection{Luminosity and absorption change}

The $0.1-10 \mathrm{keV}$ X-ray emission observed from Nova LMC 2000 at 17 and 51 days after the explosion is well described by a bremsstrahlung or Raymond-Smith model. Spectral fitting reveals that during the first observation the absorbing column was much larger, indicating intrinsic absorption due to the ejected shell. This is independent of the model used in the fitting. Despite the smaller count rate in the first observation with respect to the second, the derived luminosity was largest in the first observation.

\subsection{The mass of the ejected envelope}

The large excess absorption determined from the first observation can be used to estimate the mass of the ejected shell, assuming no clumpiness (and a filling factor of 1 though this is likely an overestimate), i.e. that the measured column density is representative for all spatial directions around the nova system. At the time of the first observation, the expansion velocity was $2000 \mathrm{~km} \mathrm{~s}^{-1}$ (Duerbeck \& Pompei 2000; Hearnshaw \& Yan Tse 2000), and thus the shell radius was $R \sim 3 \times 10^{9} \mathrm{~km}$, if no substantial deceleration has occurred during the first 17 days after the nova explosion. Using a mean column density of neutral hydrogen of $5 \times 10^{22} \mathrm{~cm}^{-2}$ (see Table 2), a mass of the shell of $\Delta M_{\mathrm{ej}}=7.5 \times 10^{-5} M_{\odot}$ is obtained. As a kind of consistency check one can also consider the later decrease of the absorbing column between the first and second XMM-Newton observation. Since the time difference, and thus the radius difference, is a factor of 3 , the absorbing column should have reduced by a factor of 9 if the shell had expanded with constant velocity. This is, within the errors, consistent with the measured change in column density (see Table 2).

An independent estimate of the ejected shell mass can be derived from the measured decay time $t_{2}$ and the relation $\log \Delta M_{\mathrm{ej}}=0.274 \times \log t_{2}-4.355$ (Della Valle et al. 2002; note that their formula as well as their figure miss a factor of $10^{-5}$ in $\left.\Delta M_{\mathrm{ej}}\right)$. This yields $\Delta M_{\mathrm{ej}}=8 \times 10^{-5} M_{\odot}$, in $\operatorname{good}$ 


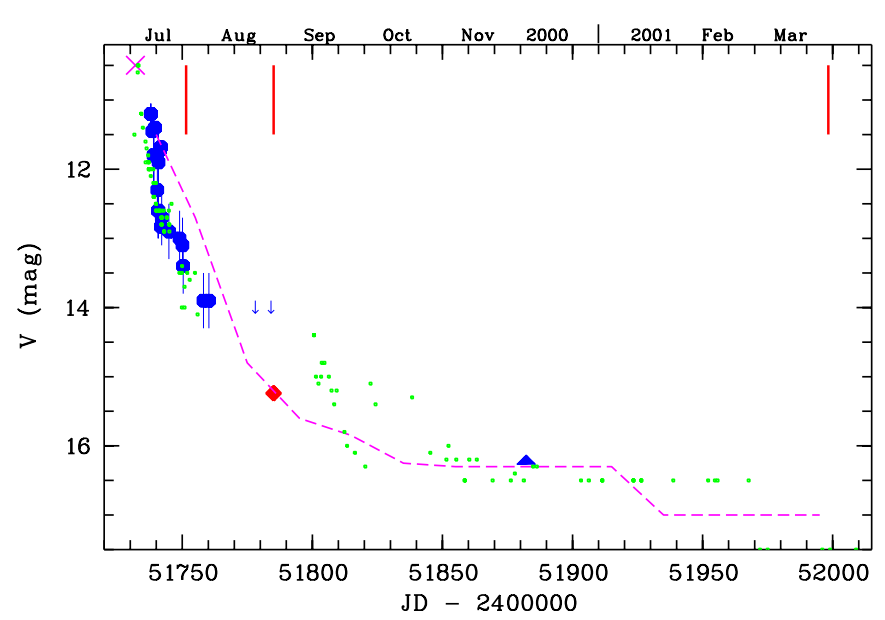

Fig. 3. Light curve of Nova LMC 2000 as measured by various observers and provided to VSNET (filled circles). Our ground-based observation from Dec. 2000 is marked by a filled triangle, and the XMM/OM observation in August 2000 by a filled lozenge. The cross marks the possible maximum as determined by a backwards extrapolation (see introduction). Vertical dashes at the top mark the times of the XMM-Newton observations. The dashed line visualizes the decay curve of the recurrent nova CI Aql (see Greiner \& Di Stefano 2002), scaled to the LMC distance. While the early decay of N LMC 2000 was substantially faster, the optical brightness in Dec. 2000 is consistent with the nova still being in the plateau phase. For comparison purposes also overplotted (with small dots) is the VSNET light curve of nova V1494 Aql (scaled in brightness) which has very similar $t_{2}$ and $t_{3}$ times.

agreement with the above estimate from the measured column density (note though that the Della Valle et al. relation refers to the ionized hydrogen mass).

Based on the relation between decay time vs. absolute magnitude (Della Valle \& Livio 1995) and our measured $t_{2}$ value, the absolute magnitude of Nova LMC 2000 would be $M_{\mathrm{V}}=$ -8.74 mag, half a magnitude brighter than the backwards extrapolation based on the spectral appearance. This would imply that this extrapolation is reasonable.

\subsection{The supersoft $X$-ray phase in novae}

No supersoft X-ray phase has been found for Nova LMC 2000: while on 25/26 July 2000 the ejected shell was still too optically thick to allow soft X-rays to penetrate, the shell had thinned considerably over the next 4 weeks (until Aug. 28) to allow the detection of supersoft emission, if it had been present. Thus, the supersoft X-ray phase in Nova LMC 2000 must have been shorter than 7 weeks, or $6 t_{2}$. In view of the fact that an HST STIS spectrum taken Aug. 19/20, i.e. 9 days before the second XMM-Newton observation shows a 1150-3120 ̊ flux of $1.6 \times 10^{38} \mathrm{erg} \mathrm{s}^{-1}$ (Shore et al. 2000), the shell burning should either has switched off during those 9 days, or the effective temperature was below $\sim 10 \mathrm{eV}$ in order not to be observable in our XMM-Newton exposure.

In an attempt to understand the reason for the largely varying duration of the supersoft X-ray phase in different novae we have compiled some information on novae with observed supersoft X-ray phase or very constraining observational limits (Table 4).

The detectability of supersoft X-ray emission from novae depends on several factors, the three most important ones (probably) being the existence/duration of such a supersoft $\mathrm{X}$-ray phase in the first place, the amount of matter ejected during the thermonuclear runaway and that blown away later during a wind phase, both of which hide the supersoft X-ray emission during the early phase after a nova until the shell has become optically thin. It is therefore interesting to ask how these factors relate to other observable parameters of a nova.

The ignition of the thermonuclear runaway of accreted hydrogen is primarily determined by the pressure at the base of the accreted envelope, which in turn depends on the mass and radius of the white dwarf and the mass of the envelope (Fujimoto 1982a). It has been argued that the $t_{3}$-time primarily depends on the mass of the white dwarf (Eq. (12) in Livio 1992). Furthermore, since also the maximum absolute magnitude of a nova correlates with the mass of the white dwarf as well as the decline rate, Della Valle et al. (2002) have recently found a correlation between the shell mass and the $t_{2}$-time (note that Shore 2002 has proposed an alternative relation using the same quantities). If the duration of the supersoft X-ray phase depended on the amount of mass which is left over after the ejection of the shell, i.e. the difference between the mass necessary to ignite the thermonuclear explosion and the mass of the matter ejected into the expanding shell, it could be expected that the supersoft X-ray phase would correlate with the $t_{2}$ - or $t_{3}$-time. We have collected the relevant data (see Table 4) for the five novae with detected supersoft X-ray emission, plus a few cases with stringent limits (including the present case Nova LMC 2000). Comparing the decay times (Cols. 2 and 3) and the implied envelope mass (Col. 4) according to Della Valle et al. (2002) with the duration of the supersoft X-ray phase (Col. 8) does not show any obvious correlation, however (though we note that envelope mass estimates are typically very uncertain). Thus, the supersoft X-ray phase is seemingly not directly correlated to the brightness decay rate and/or the mass of the white dwarf. This also argues against the simple scenario of Truran \& Glasner (1995) and Vanlandingham et al. (2001): for instance, N LMC 1995 with a rather short decline time and a white dwarf mass of $\sim 1.2 M_{\odot}$ according to the $t_{3}-M_{\mathrm{WD}}$ relation would have an expected supersoft X-ray phase of less than $1 \mathrm{yr}$, but is observed as supersoft X-ray source for over 6 years now (Orio et al. 2003).

We have also considered the expansion velocities of the nova shells (Col. 6 in Table 4), since it is proportional to the pressure at the base of the accreted shell. There is an interesting correlation with the duration of the supersoft X-ray phase in 8 sources except for Nova LMC 2000 which falls off completely (Fig. 4). Thus, either this correlation is chance coincidence due to the small number statistics, or the non-detection the supersoft X-ray phase is caused by a much higher than estimated absorbing column for which we have no other evidence. One could imagine that the (hard) X-ray emission which is seen on 28 August 2000 originates outside the expelled envelope, and therefore the small absorbing column derived from the fit of that emission is not representative of the column which 
Table 4. Details on novae with detected supersoft X-ray emission (top 5 lines), N LMC 2000, and recent recurrent novae (RN; last three lines).

\begin{tabular}{|c|c|c|c|c|c|c|c|c|c|}
\hline Nova Name & $\begin{array}{c}t_{2} \\
\text { (days) }\end{array}$ & $\begin{array}{c}t_{3} \\
\text { (days) }\end{array}$ & $\begin{array}{c}M_{\mathrm{WD}} \\
\left(M_{\odot}\right)^{(a)}\end{array}$ & $\underset{(b)}{\log \Delta M_{\mathrm{ej}}}$ & $\begin{array}{c}V_{\text {expa }}{ }^{(c)} \\
\left(\mathrm{km} \mathrm{s}^{-1}\right)\end{array}$ & $\begin{array}{c}P_{\text {orb }} \\
\text { (days) }\end{array}$ & $\log \Delta M_{\mathrm{ign}}$ & Limits on supersoft phase & $\begin{array}{l}\text { Refs. } \\
\text { (d) }\end{array}$ \\
\hline GQ Mus & 23.0 & 45 & 0.87 & -3.98 & 800 & 0.0593 & -3.4 & $10 \mathrm{yrs}$ & $1-3$ \\
\hline N Cyg 1992 & 16.0 & 42 & 0.90 & -4.02 & 2000 & 0.0812 & -3.8 & turnoff at days $32-38 \times t_{2}$ & $4-6$ \\
\hline N LMC 1995 & $12^{(e)}$ & $16^{(e)}$ & 1.23 & -4.22 & 900 & - & & $>6$ yrs and continuing & $7-9$ \\
\hline V382 Vel & 4.5 & 9 & 1.17 & -4.18 & 2700 & 0.1461 & -4.8 & at $44 \times t_{2}$; none at $60 \times t_{2}$ & 10 \\
\hline V1494 Aql & $6.6^{(f)}$ & $16^{(f)}$ & 1.09 & -4.13 & 2800 & 0.1346 & -4.6 & none $\lesssim 28 \times t_{2}$, but at $38-45 \times t_{2}$ & 11,12 \\
\hline N LMC 2000 & 9.0 & 22 & 1.02 & -4.09 & 2200 & - & & less than $5.5 \times t_{2}$ & \\
\hline U Sco $(\mathrm{RN})$ & 5.0 & 7 & 1.20 & $-5.99^{(g)}$ & 4500 & 1.230 & -5.3 & at $4 \times t_{2}$ & 13 \\
\hline IM Nor (RN) & $20.5^{(b)}$ & $47^{(b)}$ & 0.85 & -3.99 & 1150 & - & & none at $1.1 \times t_{2}$ & 12,14 \\
\hline CI Aql (RN) & 30.0 & 36 & 0.94 & -3.95 & 2400 & 0.6184 & -5.2 & less than $14 \times t_{2}$ & 15,16 \\
\hline
\end{tabular}

(a) According to the $t_{3}-M_{\mathrm{WD}}$ relation (Livio 1992).

(b) According to $\log \Delta M_{\mathrm{ej}}=0.274 \times \log t_{2}-4.355$ (Della Valle et al. 2002).

(c) Usually derived for the $\mathrm{H} \alpha$ line. For V382 Vel, lacking an estimate based on $\mathrm{H} \alpha$, we used the number given for UV lines and divide by a factor 2 (Shore 2002).

(d) References: (1) Ögelman et al. (1993), (2) Pequignot et al. (1993), (3) Shanley et al. (1995), (4) Krautter et al. (1996), (5) Chochol et al. (1993), (6) Balman et al. (1998), (7) Della Valle et al. (1995), (8) Orio \& Greiner (1999), (9) Orio et al. (1993), (10) Orio et al. (2002), (11) Venturini et al. (2000), (12) Starrfield et al. (2002), (13) Iijima (2002), (14) Duerbeck et al. (2002), (15) Greiner \& Di Stefano (2002), (16) Kiss et al. (2001).

(d) Determined here from the VSNET light curves.

${ }^{(g)}$ Instead of the prediction we used the value as measured by Della Valle et al. (2002).

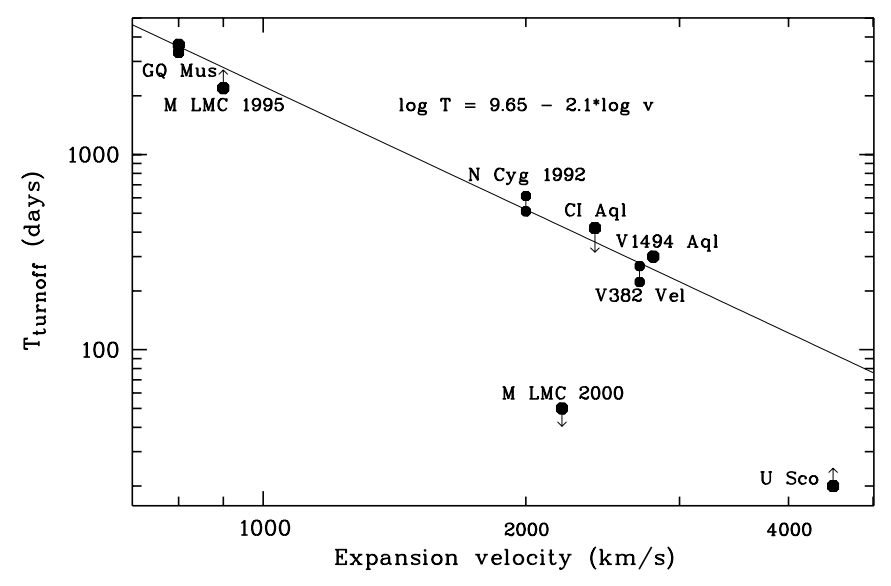

Fig. 4. Correlation between the envelope expansion velocity and the turn-off times of novae (see Table 4). For GQ Mus, Nova Cyg 1992 and V382 Vel two points are plotted, resembling the temporal limits between observed supersoft X-ray emission and $\mathrm{H}$ burning switch-off. For CI Aql and U Sco only limits are available. Nova LMC 2000 does not fit into this correlation unless the intrinsic absorbing column for the supersoft X-ray emission has been muc larger than estimated in Sects. 2 and 3. If the relation were true, one would predict that IM Nor with its rather small expansion velocity would turn into a long-lived ( $\sim 3$ yrs) supersoft X-ray phase.

blocks the supersoft emission from the white dwarf. A third alternative is that the burning continued much longer, but at such low temperature $(<10 \mathrm{eV})$ that made it impossible to be detected by XMM-Newton.
A somewhat surprising correlation is found when plotting the orbital period over the X-ray turn-off time (Fig. 5): systems with a short orbital period have a long $\mathrm{H}$ shell burning period. One possible explanation would be the higher irradiation of the companion star in short orbital period binaries, which in turn may increase the mass transfer rate to the white dwarf. But even without considering irradiation, the orbital period is strongly correlated to the mass transfer rate in cataclysmic binaries (e.g. Patterson 1984), and therefore can be expected to be a dominant factor in the evolution of a nova through a supersoft X-ray phase (see below).

A different way to consider the problem of the supersoft $\mathrm{X}$-ray phase duration is to remember that the $\mathrm{H}$ shell burning may cease before all material is consumed. As has first been argued by Fujimoto (1982b), burning will cease when the mass of the shell becomes less than a critical value $M_{\mathrm{ext}}$, below which the temperature at the bottom of the envelope is too low for the shell burning to compensate the energy loss from the surface. Thus, the important factor is not the absolute value of the matter left after the shell has been expelled, but the difference of this left-over matter and the critical mass $M_{\text {ext }}$. In evaluating this suggested correlation (Fujimoto 1982b), we have (i) derived the accretion rate from the orbital period using the "master equation of binary evolution" for the case of rotational braking (Eq. (38) in Patterson 1984), (ii) deduced the white dwarf mass from the $t_{3}$ time (Eq. (13) in Livio 1992), (iii) by using the accretion rate and white dwarf masses determined the mass $\left(\Delta M_{\text {ign }}\right)$ necessary to ignite the shell burning depending on the white dwarf mass and accretion rate (Fig. 7 


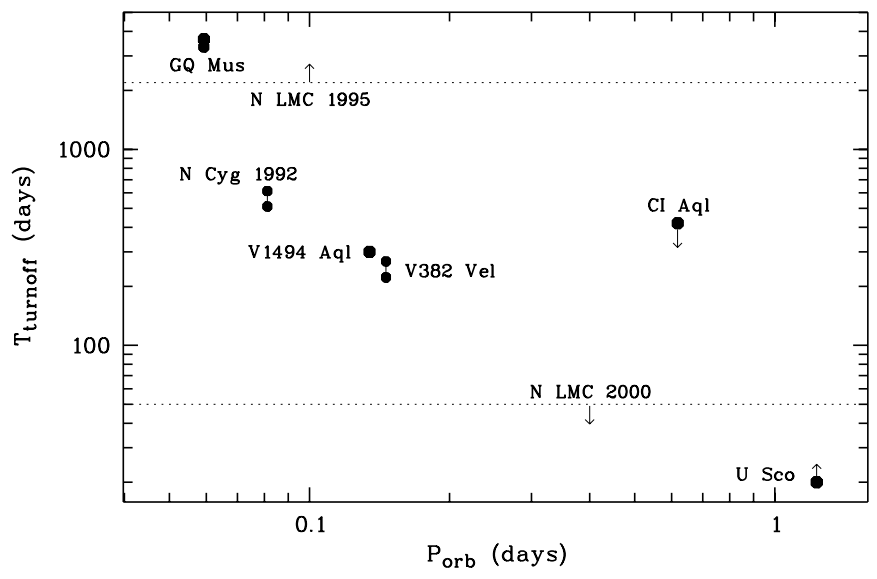

Fig. 5. Correlation between the orbital periods and the turn-off times of novae (see Table 4). Symbols and limits as in Fig. 4.

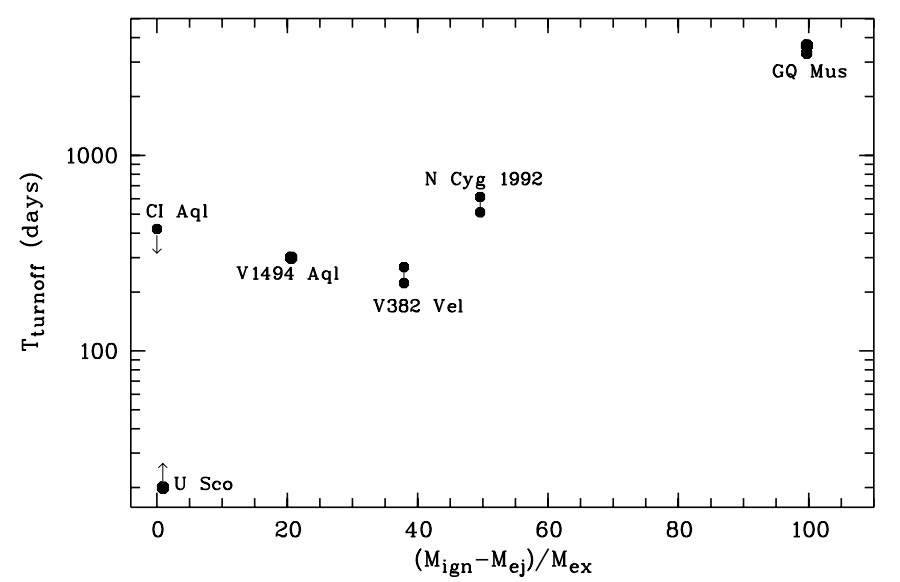

Fig. 6. Correlation between the turn-off times of novae (see Table 4) and the ratio of left-over mass to the critical mass where $\mathrm{H}$ shell burning will cease. Symbols and limits as in Fig. 4.

from Fujimoto 1982b; see Col. 8 in Table 4), (iv) derived the ejected shell mass from the $t_{2}$ time (Della Valle et al. 2002; see Col. 5 in Table 4), (v) compared the difference of the total shell mass $\left(\Delta M_{\mathrm{ign}}\right)$ and the ejected mass $\left(\Delta M_{\mathrm{ej}}\right)$ with the critical mass $\left(\Delta M_{\text {ex }}\right)$ which varies between $\sim 5 \times 10^{-5} M_{\odot}$ for a $0.8 M_{\odot}$ white dwarf mass down to $\sim 10^{-6} M_{\odot}$ for a $1.2 M_{\odot}$ white dwarf mass.

Figure 6 shows this ratio in dependence of the X-ray turnoff time for the six novae for which the relevant data $\left(t_{2}, t_{3}\right.$, $\left.P_{\text {orb }}\right)$ are available. We find that three sources have substantial mass above the critical $\Delta M_{\mathrm{ex}}$ : GQ Mus has by far the largest mass excess, consistent with its $\sim 10$ yr supersoft X-ray phase, as well as N Cyg 1992 and V1494 Aql. The other three systems, V382 Vel, CI Aql and U Sco happen to fall very near their $\Delta M_{\mathrm{ex}}$ values, and according to the suggestion of Fujimoto (1982b) would turn off very rapidly after the explosion. This is consistent with the observations.

Of course, there are several limitations to this simple picture, and additional factors are thought to also influence the duration of the supersoft X-ray phase, but were ignored here: (i) Metallicity is certainly an important factor which determines the appearance of supersoft X-rays. With Nova LMC 2000 having the same metallicity and additionally a very similar environment (less than 20 arcmin offset) as the supersoft Nova LMC 1995 (Orio \& Greiner 1999), one could have hoped for a similar X-ray behaviour. But obviously the limits on the supersoft phase in Nova LMC 2000 imply a much shorter duration than the more than 6 years (and continuing) of Nova LMC 1995 (Orio et al. 2003). (ii) It is generally agreed upon that there is a continuous, long-lasting loss of matter (wind) after the possible ejection at the time of the thermonuclear runaway. Wind-driven mass loss is expected to be strongly mass dependent, i.e. the radiation pressure grows for larger white dwarf mass. This would imply a shorter supersoft X-ray phase for novae with larger white dwarf masses (e.g. Starrfield et al. 1991; Yungelson et al. 1996).

Unfortunately, no statement can be made concerning N LMC 2000. It would be interesting to determine the orbital periods of N LMC 2000 and N LMC 1995 to determine their location relative to $\Delta M_{\mathrm{ex}}$, and thus to test the above hypothesis that the duration of the supersoft X-ray phase is determined by the ratio of the left-over mass to the critical mass $\Delta M_{\mathrm{ex}}$ for shell burning. If the above relations hold, we would infer orbital periods in the range of $0.5-1$ day for Nova LMC 2000 and $2 \mathrm{~h}$ for Nova LMC 1995.

Acknowledgements. We are highly indebted to F. Jansen for granting XMM-Newton Director's discretionary time for these Target of Opportunity observations of Nova LMC 2000. We are grateful to the VSNET observers for providing most of the measurements plotted in Fig. 3. JG thanks I. Bond for the details of the early photometry obtained within the MOA project (www.vuw.ac.nz/scps/moa), as well as C. James and A. Breeveld for the help in the attempt to make use of the OM grism data. Based on observations obtained with XMM-Newton, an ESA science mission with instruments and contributions directly funded by ESA Member States and NASA.

\section{References}

Balman, S., Krautter, J., \& Ögelman, H. 1998, ApJ, 499, 395

Bond, I. A., \& Kilmartin, P. M. 2000, IAUC, 7457

Chochol, D., Hric, L., Urban, Z., et al. 1993, A\&A, 277, 103

Duerbeck, H. W., \& Pompei, E. 2000, IAUC, 7457

Duerbeck, H. W., Baptista, R., Dutra, C. M., \& Sterken, C. 2002, IAUC, 7799

Della Valle, M., \& Livio, M. 1995, ApJ, 452, 704

Della Valle, M., Masetti, N., \& Benetti, S. 1995, IAUC, 6144

Della Valle, M., Pasquini, L., Daou, D., \& Williams, R. E. 2002, A\&A, 390, 155

Fujimoto, M. Y. 1982a, ApJ, 257, 752

Fujimoto, M. Y. 1982b, ApJ, 257, 767

Greiner, J., \& DiStefano, R. 2002, ApJ, 578, L59

Hearnshaw, J. B., \& Yan Tse, J. 2000, IAUC, 7457

Iijima, T. 2002, A\&A, 387, 1013

Kiss, L. L., Thomson, J. R., Ogloza, W., Füresz, G., \& Sziladi, K. 2001, A\&A, 366, 858

Krautter, J., Ögelman, H., Starrfield, S., Wichmann, R., \& Pfeffermann, E. 1996, ApJ, 456, 788

Liller, W. 2000, IAUC, 7453 
Livio, M. 1992, ApJ, 393, 516

Luks, T. 1994, RvMA 7, 171

MacDonald, J., Fujimoto, M. Y., \& Truran, J. W. 1985, ApJ, 294, 263

Mason, K. O., Breeveld, A., Much, R., et al. 2001, A\&A, 365, L36

Ögelman, H., Orio, M., Krautter, J., \& Starrfield, S. 1993, Nature, 361, 331

Orio, M., \& Greiner, J. 1999, A\&A, 344, L13

Orio, M., Parmar, A. N., Greiner, J., et al. 2002, MN 333, L11

Orio, M., Hartmann, W., Still, M., \& Greiner, J. 2003, ApJ, in press

Patterson, J. 1984, ApJS, 54, 443

Pequignot, D., Petitjean, P., Boisson, C., \& Krautter, J. 1993, A\&A, 271,219

Shanley, L., Ögelman, H., Gallagher, J. S., Orio, M., \& Krautter, J. 1995, ApJ, 438, L95

Shore, S. N., Starrfield, S., Bond, H. E., et al. 2000, IAUC, 7486

Shore, S. N. 2002, in Classical Nova Explosions, ed. M. Hernanz, \& J. José, AIP Conf. Proc., 637, 175
Starrfield, S., Truran, J. W., Sparks, W. M., \& Krautter, J. 1991, in Extreme Ultraviolet Astronomy, ed. R. F. Malina, \& S. Bowyer (New York: Pergamon), 168

Starrfield, S. 2002, in Classical Nova Explosions, ed. M. Hernanz, \& J. José, AIP Conf. Proc., 637, 89

Strüder, L., Briel, U., Dennerl, K., et al. 2001, A\&A, 365, L18

Truran, J. W. 2002, in The Physics of Cataclysmic Cariables and Related Objects, ed. B. T. Gänsicke, K. Beuermann, \& K. Reinsch, ASP Conf., 261, 576

Truran, J. W., \& Glasner, S. A. 1995, in Cataclysmic Variables, ed. A. Bianchini, M. Della Valle, \& M. Orio, ASSL, 205, 453

Vanlandingham, K. M., Schwarz, G. J., Shore, S. N., \& Starrfield, S. 2001, AJ, 121, 1126

Venturini, C., Rudy, R. J., Lynch, D. K., et al. 2000, IAUC, 7490

Yungelson, L., Livio, M., Truran, J. W., et al. 1996, ApJ, 466, 890 